\title{
Strong convergence theorems for a class of split feasibility problems and fixed point problem in Hilbert spaces
}

Jinhua Zhu' ${ }^{1}$, Jinfang Tang ${ }^{1}$ and Shih-sen Chang ${ }^{2 *}$

*Correspondence:

changss2013@163.com

${ }^{2}$ Center for General Education,

China Medical University, Taichung,

Taiwan

Full list of author information is

available at the end of the article

\begin{abstract}
In this paper we consider a class of split feasibility problem by focusing on the solution sets of two important problems in the setting of Hilbert spaces. One of them is the set of zero points of the sum of two monotone operators and the other is the set of fixed points of mappings. By using the modified forward-backward splitting method, we propose a viscosity iterative algorithm. Under suitable conditions, some strong convergence theorems of the sequence generated by the algorithm to a common solution of the problem are proved. At the end of the paper, some applications and the constructed algorithm are also discussed.
\end{abstract}

MSC: $26 \mathrm{~A} 18 ; 47 \mathrm{H} 04 ; 47 \mathrm{H} 05 ; 47 \mathrm{H} 10$

Keywords: Split feasibility; Maximal monotone operators; Inverse strongly monotone operator; Fixed point problems; Strong convergence theorems

\section{Introduction}

Many applications of the split feasibility problem (SFP), which was first introduced by Censor and Elfving [1], have appeared in various fields of science and technology, such as in signal processing, medical image reconstruction and intensity-modulated radiation therapy (for more information, see [2,3] and the references therein). In fact, Censor and Elfving [1] studied SFP in a finite-dimensional space, by considering the problem of finding a point

$$
x^{*} \in C \text { such that } A x^{*} \in Q \text {, }
$$

where $C$ and $Q$ are nonempty closed convex subsets of $\mathbb{R}^{n}$, and $A$ is an $n \times n$ matrix. They introduced an iterative method for solving SFP.

On the other hand, variational inclusion problems are being used as mathematical programming models to study a large number of optimization problems arising in finance, economics, network, transportation and engineering science. The formal form of a variational inclusion problem is the problem of finding $x^{*} \in H$ such that

$$
0 \in B x^{*},
$$

(c) The Author(s) 2018. This article is distributed under the terms of the Creative Commons Attribution 4.0 International License (http://creativecommons.org/licenses/by/4.0/), which permits unrestricted use, distribution, and reproduction in any medium, provided you give appropriate credit to the original author(s) and the source, provide a link to the Creative Commons license, and indicate if changes were made. 
where $B: H \rightarrow 2^{H}$ is a set-valued operator. If $B$ is a maximal monotone operator, the elements in the solution set of problem (1.2) are called the zeros of this maximal monotone operator. This problem was introduced by Martinet [4], and later it has been studied by many authors. It is well known that the popular iteration method that was used for solving problem (1.2) is the following proximal point algorithm: for a given $x \in H$,

$$
x_{n+1}=J_{\lambda_{n}}^{B} x_{n}, \quad \forall n \in \mathbb{N}
$$

where $\left\{\lambda_{n}\right\} \subset(0, \infty)$ and $J_{\lambda_{n}}^{B}=\left(I+\lambda_{n} B\right)^{-1}$ is the resolvent of the considered maximal monotone operator $B$ corresponding to $\lambda_{n}$ (see, also [5-9] for more details).

In view of SFP and the fixed point problem, very recently, Montira et al. [10] considered the problem of finding a point $x^{*} \in H$ such

$$
0 \in A x^{*}+B x^{*} \quad \text { and } L x^{*} \in F(T)
$$

where $A: H_{1} \rightarrow H_{1}$ is a monotone operator, and $B: H_{1} \rightarrow 2^{H_{1}}$ is a maximal monotone operator, $L: H_{1} \rightarrow H_{2}$ is a bounded linear operator and $T: H_{2} \rightarrow H_{2}$ is a nonexpansive mapping.

They considered the following iterative algorithm: for any $x_{0} \in H_{1}$,

$$
x_{n+1}=J_{\lambda_{n}}^{B}\left(\left(I-\lambda_{n} A\right)-\gamma_{n} L^{*}(I-T) L\right) x_{n}, \quad \forall n \in \mathbb{N},
$$

where $\left\{\lambda_{n}\right\}$ and $\left\{\gamma_{n}\right\}$ satisfy some suitable control conditions, and $J_{\lambda_{n}}^{B}$ is the resolvent of a maximal monotone operator $B$ associated to $\lambda_{n}$, and proved that sequence (1.4) weakly converges to a point $x^{*} \in \Omega_{L, T}^{A+B}$, where $\Omega_{L, T}^{A+B}$ is the solution set of problem (1.3).

Motivated by the work of Montira et al. [10] and the research in this direction, the purpose of this paper is to study the following split feasibility problem and fixed point problem: find $x^{*} \in H$ such that

$$
0 \in A x^{*}+B x^{*}, \quad L x^{*} \in F(T) \quad \text { and } \quad x^{*} \in F(S),
$$

where $A, B, L$ are the same as in (1.3) and $S: H_{1} \rightarrow H_{1}$ is a nonexpansive mapping. By using a modified forward-backward splitting method, we propose a viscosity iterative algorithm (see (3.4) below). Under suitable conditions, some strong convergence theorems of the sequence generated by the algorithm to a zero of the sum of two monotone operators and fixed point of mappings are proved. At the end of the paper, some applications and the constructed algorithm are also discussed. The results presented in the paper extend and improve the main results of Montira et al. [10], Byrne et al. [11], Takahashi et al. [12] and Passty [13].

\section{Preliminaries}

Throughout this paper, we denote by $\mathbb{N}$ the set of positive integers, and by $\mathbb{R}$ the set of real numbers. Let $H$ be a real Hilbert space with the inner product $\langle\cdot, \cdot\rangle$ and norm $\|\cdot\|$, respectively. When $\left\{x_{n}\right\}$ is a sequence in $H$, we denote the weak convergence of $\left\{x_{n}\right\}$ to $x$ in $H$ by $x_{n} \rightarrow x$. 
Let $T: H \rightarrow H$ be a mapping. We say that $T$ is a Lipschitz mapping if there exists an $L>0$ such that

$$
\|T x-T y\| \leq L\|x-y\|, \quad \forall x, y \in H .
$$

The number $L$, associated with $T$, is called a Lipschitz constant. If $L=1$, we say that $T$ is a nonexpansive mapping, that is,

$$
\|T x-T y\| \leq\|x-y\|, \quad \forall x, y \in H .
$$

We say that $T$ is firmly nonexpansive if

$$
\langle T x-T y, x-y\rangle \geq\|T x-T y\|^{2}, \quad \forall x, y \in H .
$$

A mapping $T: H \rightarrow H$ is said to be an averaged mapping if it can be written as the average of the identity $I$ and a nonexpansive mapping, that is,

$$
T=(1-\alpha) I+\alpha S
$$

where $\alpha \in(0,1)$ and $S: H \rightarrow H$ is a nonexpansive mapping [14]. More precisely, when (2.1) holds, we say that $T$ is $\alpha$-averaged. It should be observed that a mapping is firmly nonexpansive if and only if it is a $\frac{1}{2}$-averaged mapping.

Let $A: H \rightarrow H$ be a single-valued mapping. For a positive real number $\beta$, we say that $A$ is $\beta$-inverse strongly monotone $(\beta$-ism) if

$$
\langle A x-A y, x-y\rangle \geq \beta\|A x-A y\|^{2}, \quad \forall x, y \in H .
$$

We now collect some important conclusions and properties, which will be needed in proving our main results.

Lemma 2.1 ([15, 16]) The following conclusions hold:

(i) The composition of finitely many averaged mappings is averaged. In particular, if $T_{i}$ is $\alpha_{i}$-averaged, where $\alpha_{i} \in(0,1)$ for $i=1,2$, then the composition $T_{1} T_{2}$ is $\alpha$-averaged, where $\alpha=\alpha_{1}+\alpha_{2}-\alpha_{1} \alpha_{2}$.

(ii) If $A$ is $\beta$-ism and $\gamma \in(0, \beta]$, then $T:=I-\gamma A$ is firmly nonexpansive.

(iii) A mapping $T: H \rightarrow H$ is nonexpansive if and only if $I-T$ is $\frac{1}{2}$-ism.

(iv) If $A$ is $\beta$-ism, then, for $\gamma>0, \gamma A$ is $\frac{\beta}{\gamma}$-ism.

(v) $T$ is averaged if and only if the complement $I-T$ is $\beta$-ism for some $\beta>\frac{1}{2}$. Indeed, for $\alpha \in(0,1), T$ is $\alpha$-averaged if and only if $I-T$ is $\frac{1}{2 \alpha}$-ism.

Lemma 2.2 ([17]) Let $T=(1-\alpha) A+\alpha N$ for some $\alpha \in(0,1)$. If $A$ is $\beta$-averaged and $N$ is nonexpansive then $T$ is $\alpha+(1-\alpha) \beta$-averaged.

Let $B: H \rightarrow 2^{H}$ be a set-valued mapping. The effective domain of $B$ is denoted by $D(B)$, that is, $D(B)=\{x \in H: B x \neq \emptyset\}$. Recall that $B$ is said to be monotone if

$$
\langle x-y, u-v\rangle \geq 0, \quad \forall x, y \in D(B), u \in B x, v \in B y .
$$


A monotone mapping $B$ is said to be maximal if its graph is not properly contained in the graph of any other monotone operator. For a maximal monotone operator $B: H \rightarrow 2^{H}$ and $r>0$, its resolvent $J_{r}^{B}$ is defined by

$$
J_{r}^{B}:=(I+r B)^{-1}: H \rightarrow D(B) .
$$

It is well known that, if $B$ is a maximal monotone operator and $r$ is a positive number, then the resolvent $J_{r}^{B}$ is single-valued and firmly nonexpansive, and $F\left(J_{r}^{B}\right)=B^{-1} 0 \equiv\{x \in H: 0 \in$ $B x\}, \forall r>0$ (see $[12,18,19])$.

Lemma 2.3 ([20]) Let $H$ be a Hilbert space and let $B$ be a maximal monotone operator on $H$. Then for all $s, t>0$ and $x \in H$,

$$
\begin{aligned}
& \frac{s-t}{s}\left\langle J_{s} x-J_{t} x, J_{s} x-x\right\rangle \geq\left\|J_{s} x-J_{t} x\right\|^{2} ; \\
& \left\|J_{s} x-J_{t} x\right\| \leq(|s-t| / s)\left\|x-J_{s} x\right\| .
\end{aligned}
$$

Lemma 2.4 ([12]) Let $H_{1}$ and $H_{2}$ be Hilbert spaces. Let $L: H_{1} \rightarrow H_{2}$ be a nonzero bounded linear operator and $\mathrm{T}: \mathrm{H}_{2} \rightarrow \mathrm{H}_{2}$ be a nonexpansive mapping. If $B: H_{1} \rightarrow 2^{H_{1}}$ is a maximal monotone operator, then

(i) $L^{*}(I-T) L$ is $\frac{1}{2\|L\|^{2}}$-ism,

(ii) For $0<r<\frac{1}{\|L\|}$,

(iia) $I-r L^{*}(I-T) L$ is $r\|L\|^{2}$-averaged,

(iib) $J_{\lambda}^{B}\left(I-r L^{*}(I-T) L\right)$ is $\frac{1+r\|L\|^{2}}{2}$-averaged, for $\lambda>0$,

(iii) If $r=\|L\|^{-2}$, then $I-r L^{*}(I-T) L$ is nonexpansive.

Lemma 2.5 ([21]) Let $B: H \rightarrow 2^{H}$ be a maximal monotone operator with the resolvent $J_{\lambda}^{B}=(I+\lambda B)^{-1}$ for $\lambda>0$. Then we have the following resolvent identity:

$$
J_{\lambda}^{B} x=J_{\mu}^{B}\left(\frac{\mu}{\lambda} x+\left(1-\frac{\mu}{\lambda}\right) J_{\lambda}^{B} x\right)
$$

for all $\mu>0$ and $x \in H$.

Lemma 2.6 ([22]) Let $C$ be a closed convex subset of a Hilbert space $H$ and let $T$ be a nonexpansive mapping of $C$ into itself. Then $U:=I-T$ is demiclosed, i.e., $x_{n} \rightarrow x_{0}$ and $U x_{n} \rightarrow y_{0}$ imply $U x_{0}=y_{0}$.

Lemma 2.7 ([10]) Let $H_{1}$ and $H_{2}$ be Hilbert spaces. Let $A: H_{1} \rightarrow H_{1}$ be a $\beta$-ism, $B: H_{1} \rightarrow$ $2^{H_{1}}$ a maximal monotone operator, $T: H_{2} \rightarrow H_{2}$ a nonexpansive mapping and $L: H_{1} \rightarrow H_{2}$ a bounded linear operator. If $\Omega_{L, T}^{A+B} \neq \emptyset$, then the following are equivalent:

(i) $z \in \Omega_{L, T}^{A+B}$,

(ii) $z=J_{\lambda}^{B}\left(\left(I_{\lambda}-A\right)-\gamma L^{*}(I-T) L\right) z$,

(iii) $0 \in L^{*}(I-T) L z+(A+B) z$,

where $\lambda, \gamma>0$ and $z \in H_{1}$.

Lemma 2.8 ([23]) Let $\left\{a_{n}\right\}$ be a sequence of nonnegative real numbers such that

$$
a_{n+1} \leq\left(1-\beta_{n}\right) a_{n}+\delta_{n}, \quad \forall n \geq 0,
$$


where $\left\{\beta_{n}\right\}$ is a sequence in $(0,1)$ and $\left\{\delta_{n}\right\}$ is a sequence in $\mathbb{R}$ such that

(i) $\sum_{n=1}^{\infty} \beta_{n}=\infty$;

(ii) $\lim \sup _{n \rightarrow \infty} \frac{\delta_{n}}{\beta_{n}} \leq 0$ or $\sum_{n=1}^{\infty}\left|\delta_{n}\right|<\infty$.

Then $\lim _{n \rightarrow \infty} a_{n}=0$.

\section{Main results}

We are now in a position to give the main result of this paper.

Lemma 3.1 Let $H_{1}$ and $H_{2}$ be two real Hilbert spaces. Let $A: H_{1} \rightarrow H_{1}$ be a $\beta$-ism, $B$ : $H_{1} \rightarrow 2^{H_{1}}$ be a maximal monotone operator, $T: H_{2} \rightarrow H_{2}$ be a nonexpansive mapping, and $L: H_{1} \rightarrow H_{2}$ be a bounded linear operator. Let $S: H_{1} \rightarrow H_{1}$ be a nonexpansive mapping such that $F(S) \cap \Omega_{L, T}^{A+B} \neq \emptyset$, where

$$
\Omega_{L, T}^{A+B}:=\left\{x \in(A+B)^{-1}(0) \cap L^{-1} F(T)\right\}
$$

is the set of solutions of problem (1.3). Let $f: H_{1} \rightarrow H_{1}$ be a contraction mapping with a contractive constant $\alpha \in(0,1)$. For any $t \in(0,1]$, let $W_{t}: H_{1} \rightarrow H_{1}$ be the mapping defined by

$$
W_{t} x=t f(x)+(1-t) S\left[J_{\lambda_{n}}^{B}\left(\left(I-\lambda_{n} A\right)-\gamma_{n} L^{*}(I-T) L\right) x\right], \quad \forall x \in H_{1},
$$

where $L^{*}$ is the adjoint of $L$ and the sequences $\lambda_{n}$ and $\gamma_{n}$ satisfy the following control conditions:

(i) $0<a \leq \lambda_{n} \leq b_{1}<\frac{\beta}{2}$,

(ii) $0<a \leq \gamma_{n} \leq b_{2}<\frac{1}{2\|L\|^{2}}$, for some $a, b_{1}, b_{2} \in \mathbb{R}$.

Then $W_{t}$ is a contraction mapping with a contractive constant $[1-t(1-\alpha)]$. Therefore $W_{t}$ has a unique fixed point for each $t \in(0,1)$.

Proof Note that, for each $n \in \mathbb{N}$, we have

$$
\left(I-\lambda_{n} A\right)-\gamma_{n} L^{*}(I-L) L=\frac{1}{2}\left(I-2 \lambda_{n} A\right)+\frac{1}{2}\left(I-2 \gamma_{n} L^{*}(I-T) L\right) .
$$

Also, by condition (i) and Lemma 2.1(ii), we know that $I-2 \lambda_{n} A$ is a firmly nonexpansive mapping, and this implies that $I-2 \lambda_{n} A$ must be a nonexpansive mapping. On the other hand, by Lemma 2.4(iia), we know that $I-2 \gamma_{n} L^{*}(I-T) L$ is $2 \gamma_{n}\|L\|^{2}$-averaged. Thus, by condition (ii) and Lemma 2.2, we see that $\left(I-\lambda_{n} A\right)-\gamma_{n} L^{*}(I-T) L$ is $\frac{1+2 \gamma_{n}\|L\|^{2}}{2}$-averaged.

Set

$$
T_{n}:=J_{\lambda_{n}}^{B}\left(\left(I-\lambda_{n} A\right)-\gamma_{n} L^{*}(I-T) L\right), \quad \forall n \geq 1 .
$$

Since $J_{\lambda_{n}}^{B}$ is $\frac{1}{2}$-averaged, by Lemma 2.1(i) we see that $T_{n}$ is $\frac{3+2 \gamma_{n}\|L\|^{2}}{4}$-averaged and hence it is nonexpansive. Further, for any $x, y \in H_{1}$, we obtain

$$
\begin{aligned}
\left\|W_{t} x-W_{t} y\right\| & =\left\|t f(x)+(1-t) S T_{n} x-t f(y)-(1-t) S T_{n} y\right\| \\
& \leq t\|f(x)-f(y)\|+(1-t)\left\|S T_{n} x-S T_{n} y\right\|
\end{aligned}
$$




$$
\begin{aligned}
& \leq t \alpha\|x-y\|+(1-t)\|x-y\| \\
& =(1-t(1-\alpha))\|x-y\| .
\end{aligned}
$$

Since $0<1-t(1-\alpha)<1$, it follows that $W_{t}$ is a contraction mapping. Therefore, by Banach contraction principle, $W_{t}$ has a unique fixed point $x_{t}$ in $H_{1}$.

Theorem 3.2 Let $H_{1}, H_{2}, A, B, T, L, S, f$ be the same as in Lemma 3.1. For any given $x_{0} \in H_{1}$, let $\left\{u_{n}\right\}$ and $\left\{x_{n}\right\}$ be the sequences generated by

$$
\left\{\begin{array}{l}
u_{n}=J_{\lambda_{n}}^{B}\left(\left(I-\lambda_{n} A\right)-\gamma_{n} L^{*}(I-T) L\right) x_{n}, \\
x_{n+1}=\alpha_{n} f\left(x_{n}\right)+\left(1-\alpha_{n}\right) S u_{n},
\end{array} \quad \forall n \geq 0,\right.
$$

where $\left\{\alpha_{n}\right\}$ is a sequence in $(0,1)$ such that $\lim _{n \rightarrow \infty} \alpha_{n}=0, \sum_{n=0}^{\infty} \alpha_{n}=\infty$ and $\sum_{n=1}^{\infty} \mid \alpha_{n}-$ $\alpha_{n-1} \mid<\infty$ and $L^{*}$ is the adjoint of $L$.

If $F(S) \cap \Omega_{L, T}^{A+B} \neq \emptyset$ and the sequences $\left\{\lambda_{n}\right\}$ and $\left\{\gamma_{n}\right\}$ satisfy the following conditions:

(i) $0<a \leq \lambda_{n} \leq b_{1}<\frac{\beta}{2}$, and $\Sigma_{n=1}^{\infty}\left|\lambda_{n}-\lambda_{n-1}\right|<\infty$,

(ii) $0<a \leq \gamma_{n} \leq b_{2}<\frac{1}{2\|L\|^{2}}$, and $\Sigma_{n=1}^{\infty}\left|\gamma_{n}-\gamma_{n-1}\right|<\infty$, for some $a, b_{1}, b_{2} \in \mathbb{R}$,

then the sequences $\left\{u_{n}\right\}$ and $\left\{x_{n}\right\}$ both converge strongly to $z \in F(S) \cap \Omega_{L, T}^{A+B}$, where $z=$ $P_{F(S) \cap \Omega_{L, T}^{A+B}} f(z)$, i.e., $z$ is a solution of problem (1.5).

Proof Take

$$
T_{n}:=J_{\lambda_{n}}^{B}\left(\left(I-\lambda_{n} A\right)-\gamma_{n} L^{*}(I-T) L\right)
$$

for each $n \in \mathbb{N}$. By Lemma 2.7, we have $\Omega_{L, T}^{A+B}=F\left(T_{n}\right)$, for all $n \in \mathbb{N}$. Thus, for each $n \in \mathbb{N}$, we can write $x_{n+1}=\alpha_{n} f\left(x_{n}\right)+\left(1-\alpha_{n}\right) S T_{n} x_{n}$. By the proof of Lemma 3.1, we see that $T_{n}$ is $\frac{3+2 \gamma_{n}\|L\|^{2}}{4}$-averaged. Thus, for each $n \in \mathbb{N}$, we can write

$$
T_{n}=\left(1-\xi_{n}\right) I+\xi_{n} V_{n}
$$

where $\xi_{n}=\frac{3+2 \gamma_{n}\|L\|^{2}}{4}$ and $V_{n}$ is a nonexpansive mapping. Consequently, we also have $\Omega_{L, T}^{A+B}=F\left(T_{n}\right)=F\left(V_{n}\right)$, for all $n \in \mathbb{N}$. Using this fact, for each $p \in F(S) \cap \Omega_{L, T}^{A+B}$, we see that

$$
\begin{aligned}
\left\|u_{n}-p\right\|^{2} & =\left\|T_{n} x_{n}-p\right\|^{2} \\
& =\left\|\left(1-\xi_{n}\right) x_{n}+\xi_{n} V_{n} x_{n}-p\right\|^{2} \\
& =\left\|\left(1-\xi_{n}\right)\left(x_{n}-p\right)+\xi_{n}\left(V_{n} x_{n}-p\right)\right\|^{2} \\
& =\left(1-\xi_{n}\right)\left\|x_{n}-p\right\|^{2}+\xi_{n}\left\|V_{n} x_{n}-p\right\|^{2}-\xi_{n}\left(1-\xi_{n}\right)\left\|x_{n}-V_{n} x_{n}\right\|^{2} \\
& \leq\left\|x_{n}-p\right\|^{2}-\xi_{n}\left(1-\xi_{n}\right)\left\|x_{n}-V_{n} x_{n}\right\|^{2}
\end{aligned}
$$

for each $n \in \mathbb{N}$. Since $I-T_{n}=\xi_{n}\left(I-V_{n}\right)$, in view of (3.5) we get

$$
\left\|u_{n}-p\right\|^{2} \leq\left\|x_{n}-p\right\|^{2}-\left(1-\xi_{n}\right)\left\|x_{n}-T_{n} x_{n}\right\|^{2},
$$


for each $n \in \mathbb{N}$. Since $\xi_{n}=\frac{3+2 \gamma_{n}\|L\|^{2}}{4} \in\left(\frac{3}{4}, 1\right)$, we obtain

$$
\left\|u_{n}-p\right\|^{2} \leq\left\|x_{n}-p\right\|^{2}
$$

Next, we estimate

$$
\begin{aligned}
\left\|x_{n+1}-p\right\| & =\left\|\alpha_{n} f\left(x_{n}\right)+\left(1-\alpha_{n}\right) S u_{n}-p\right\| \\
& \leq \alpha_{n}\left\|f\left(x_{n}\right)-p\right\|+\left(1-\alpha_{n}\right)\left\|S u_{n}-p\right\| \\
& \leq \alpha_{n}\left(\left\|f\left(x_{n}\right)-f(p)\right\|+\|f(p)-p\|\right)+\left(1-\alpha_{n}\right)\left\|u_{n}-p\right\| \\
& \leq \alpha_{n} \alpha\left\|x_{n}-p\right\|+\alpha_{n}\|f(p)-p\|+\left(1-\alpha_{n}\right)\left\|x_{n}-p\right\| \\
& \leq\left(1-\alpha_{n}(1-\alpha)\right)\left\|x_{n}-p\right\|+\alpha_{n}\|f(p)-p\| \\
& \leq \max \left\{\left\|x_{n}-p\right\|, \frac{\|f(p)-p\|}{1-\alpha}\right\}
\end{aligned}
$$

By induction, we can prove that

$$
\left\|x_{n+1}-p\right\| \leq \max \left\{\left\|x_{0}-p\right\|, \frac{\|f(p)-p\|}{1-\alpha}\right\}, \quad \forall n \geq 0
$$

Hence $\left\{x_{n}\right\}$ is bounded and so are $\left\{u_{n}\right\},\left\{f\left(x_{n}\right)\right\}$ and $\left\{S u_{n}\right\}$.

Next, we show that

$$
\lim _{n \rightarrow \infty}\left\|x_{n+1}-x_{n}\right\|=0
$$

In fact, it follows from (3.4) that

$$
\begin{aligned}
\left\|x_{n+1}-x_{n}\right\|= & \left\|\alpha_{n} f\left(x_{n}\right)+\left(1-\alpha_{n}\right) S u_{n}-\left(\alpha_{n-1} f\left(x_{n-1}\right)+\left(1-\alpha_{n-1}\right) S u_{n-1}\right)\right\| \\
= & \| \alpha_{n} f\left(x_{n}\right)-\alpha_{n} f\left(x_{n-1}\right)+\alpha_{n} f\left(x_{n-1}\right)-\alpha_{n-1} f\left(x_{n-1}\right)+\left(1-\alpha_{n}\right) S u_{n} \\
& -\left(1-\alpha_{n}\right) S u_{n-1}+\left(1-\alpha_{n}\right) S u_{n-1}-\left(1-\alpha_{n-1}\right) S u_{n-1} \| \\
\leq & \alpha_{n} \alpha\left\|x_{n}-x_{n-1}\right\|+\left(1-\alpha_{n}\right)\left\|S u_{n}-S u_{n-1}\right\|+2\left|\alpha_{n}-\alpha_{n-1}\right| K \\
\leq & \alpha_{n} \alpha\left\|x_{n}-x_{n-1}\right\|+\left(1-\alpha_{n}\right)\left\|u_{n}-u_{n-1}\right\|+2\left|\alpha_{n}-\alpha_{n-1}\right| K,
\end{aligned}
$$

where $K:=\sup \left\{\left\|f\left(x_{n}\right)\right\|+\left\|S u_{n}\right\|: n \in \mathbb{N}\right\}$.

Put

$$
\begin{aligned}
& y_{n}=\left(\left(I-\lambda_{n} A\right)-\gamma_{n} L^{*}(I-T) L\right) x_{n} \text { and } \\
& u_{n}=T_{n} x_{n}=J_{\lambda_{n}}^{B} y_{n} .
\end{aligned}
$$

Since $J_{\lambda_{n}}^{B}\left(\left(I-\lambda_{n} A\right)-\gamma_{n} L^{*}(I-T) L\right)$ is nonexpansive, it follows from Lemma 2.3 that

$$
\begin{aligned}
\left\|u_{n+1}-u_{n}\right\| & =\left\|J_{\lambda_{n+1}}^{B} y_{n+1}-J_{\lambda_{n}}^{B} y_{n}\right\| \\
& \leq\left\|J_{\lambda_{n+1}}^{B} y_{n+1}-J_{\lambda_{n+1}}^{B}\left(\left(I-\lambda_{n+1} A\right)-\gamma_{n+1} L^{*}(I-T) L\right) x_{n}\right\|
\end{aligned}
$$




$$
\begin{aligned}
& +\left\|J_{\lambda_{n+1}}^{B}\left(\left(I-\lambda_{n+1} A\right)-\gamma_{n+1} L^{*}(I-T) L\right) x_{n}-J_{\lambda_{n}}^{B} y_{n}\right\| \\
\leq & \left\|x_{n+1}-x_{n}\right\|+\left\|J_{\lambda_{n+1}}^{B}\left(\left(I-\lambda_{n+1} A\right)-\gamma_{n+1} L^{*}(I-T) L\right) x_{n}-J_{\lambda_{n}}^{B} y_{n}\right\| \\
\leq & \| J_{\lambda_{n+1}}^{B}\left(\left(I-\lambda_{n+1} A\right)-\gamma_{n+1} L^{*}(I-T) L\right) x_{n} \\
& -J_{\lambda_{n+1}}^{B}\left(\left(I-\lambda_{n} A\right)-\gamma_{n} L^{*}(I-T) L\right) x_{n} \| \\
& +\left\|J_{\lambda_{n+1}}^{B} y_{n}-J_{\lambda_{n}}^{B} y_{n}\right\|+\left\|x_{n+1}-x_{n}\right\| \\
\leq & \left\|\left(\left(I-\lambda_{n+1} A\right)-\gamma_{n+1} L^{*}(I-T) L\right) x_{n}-\left(\left(I-\lambda_{n} A\right)-\gamma_{n} L^{*}(I-T) L\right) x_{n}\right\| \\
& +\left\|J_{\lambda_{n+1}}^{B} y_{n}-J_{\lambda_{n}}^{B} y_{n}\right\|+\left\|x_{n+1}-x_{n}\right\| \\
\leq & \left|\lambda_{n+1}-\lambda_{n}\right|\left\|A x_{n}\right\|+\left|\gamma_{n+1}-\gamma_{n}\right|\left\|L^{*}(I-T) L x_{n}\right\| \\
& +\frac{\left|\lambda_{n+1}-\lambda_{n}\right|}{a}\left\|J_{\lambda_{n+1}}^{B} y_{n}-y_{n}\right\|+\left\|x_{n+1}-x_{n}\right\| \\
\leq & \left\|x_{n+1}-x_{n}\right\|+M_{1}\left|\lambda_{n+1}-\lambda_{n}\right|+M_{2}\left|\gamma_{n+1}-\gamma_{n}\right|
\end{aligned}
$$

where $M_{1}$ and $M_{2}$ are constants defined by

$$
\begin{aligned}
& M_{1}=\sup _{n}\left(\left\|A x_{n}\right\|+\frac{1}{a}\left\|J_{\lambda_{n+1}}^{B} y_{n}-y_{n}\right\|\right), \\
& M_{2}=\sup _{n}\left\|L^{*}(I-T) L x_{n}\right\| .
\end{aligned}
$$

Therefore it follows from (3.10) and (3.11) that

$$
\left\|x_{n+1}-x_{n}\right\| \leq\left(1-\alpha_{n}(1-\alpha)\right)\left\|x_{n}-x_{n-1}\right\|+M_{1}\left|\lambda_{n+1}-\lambda_{n}\right|+M_{2}\left|\gamma_{n+1}-\gamma_{n}\right|+2\left|\alpha_{n}-\alpha_{n-1}\right| K .
$$

Take

$$
\begin{aligned}
& \beta_{n}:=\alpha_{n}(1-\alpha) \text { and } \\
& \delta_{n}:=M_{1}\left|\lambda_{n+1}-\lambda_{n}\right|+M_{2}\left|\gamma_{n+1}-\gamma_{n}\right|+2\left|\alpha_{n}-\alpha_{n-1}\right| K .
\end{aligned}
$$

It follows from Lemma 2.8 that

$$
\lim _{n \rightarrow \infty}\left\|x_{n+1}-x_{n}\right\|=0
$$

Now, we write

$$
\begin{aligned}
x_{n+1}-x_{n} & =\alpha_{n} f\left(x_{n}\right)+\left(1-\alpha_{n}\right) S u_{n}-x_{n} \\
& =\alpha_{n}\left(f\left(x_{n}\right)-x_{n}\right)+\left(1-\alpha_{n}\right)\left(S u_{n}-x_{n}\right) .
\end{aligned}
$$

Since $\left\|x_{n+1}-x_{n}\right\| \rightarrow 0$ and $\alpha_{n} \rightarrow 0$ as $n \rightarrow \infty$, we obtain

$$
\lim _{n \rightarrow \infty}\left\|S u_{n}-x_{n}\right\|=0
$$

Next, we prove that

$$
\lim _{n \rightarrow \infty}\left\|x_{n}-u_{n}\right\|=\lim _{n \rightarrow \infty}\left\|x_{n}-T_{n} x_{n}\right\|=0
$$


In fact, it follows from (3.4) and (3.6) That

$$
\begin{aligned}
\left\|x_{n+1}-p\right\|^{2} & =\left\|\alpha_{n} f\left(x_{n}\right)+\left(1-\alpha_{n}\right) S u_{n}-p\right\|^{2} \\
& \leq \alpha_{n}\left\|f\left(x_{n}\right)-p\right\|^{2}+\left(1-\alpha_{n}\right)\left\|S u_{n}-p\right\|^{2} \\
& \leq \alpha_{n}\left\|f\left(x_{n}\right)-p\right\|^{2}+\left(1-\alpha_{n}\right)\left\|u_{n}-p\right\|^{2} \\
& \leq \alpha_{n}\left\|f\left(x_{n}\right)-p\right\|^{2}+\left(1-\alpha_{n}\right)\left(\left\|x_{n}-p\right\|^{2}-\left(1-\xi_{n}\right)\left\|x_{n}-T_{n} x_{n}\right\|^{2}\right) \\
& \leq \alpha_{n}\left\|f\left(x_{n}\right)-p\right\|^{2}+\left\|x_{n}-p\right\|^{2}-\left(1-\xi_{n}\right)\left\|x_{n}-T_{n} x_{n}\right\|^{2} .
\end{aligned}
$$

Hence, we obtain

$$
\begin{aligned}
\left(1-\xi_{n}\right)\left\|x_{n}-T_{n} x_{n}\right\|^{2} & \leq \alpha_{n}\left\|f\left(x_{n}\right)-p\right\|^{2}+\left(\left\|x_{n}-p\right\|^{2}-\left\|x_{n+1}-p\right\|^{2}\right) \\
& \leq \alpha_{n}\left\|f\left(x_{n}\right)-p\right\|^{2}+\left(\left\|x_{n}-p\right\|+\left\|x_{n+1}-p\right\|\right)\left\|x_{n}-x_{n+1}\right\|
\end{aligned}
$$

Since $\alpha_{n} \rightarrow 0$ as $n \rightarrow \infty$, and $\xi_{n}=\frac{3+2 \gamma_{n}\|L\|^{2}}{4} \in\left(\frac{3}{4}, 1\right)$, from (3.12) we obtain

$$
\lim _{n \rightarrow \infty}\left\|u_{n}-x_{n}\right\|=\left\|x_{n}-T_{n} x_{n}\right\|=0
$$

Therefore we have

$$
\left\|S u_{n}-u_{n}\right\| \leq\left\|S u_{n}-x_{n}\right\|+\left\|x_{n}-u_{n}\right\| \rightarrow 0, \quad \text { as } n \rightarrow \infty \text {. }
$$

On the other hand, since $\left\{x_{n}\right\}$ is bounded, let $\left\{x_{n_{j}}\right\}$ be any subsequence of $\left\{x_{n}\right\}$ with $x_{n_{j}} \rightarrow \hat{x}$. Also, we assume that $\lambda_{n_{j}} \rightarrow \hat{\lambda} \in\left(0, \frac{\beta}{2}\right)$ and $\gamma_{n_{j}} \rightarrow \hat{\gamma} \in\left(0, \frac{1}{2\|L\|^{2}}\right)$.

Letting

$$
\hat{T}=J_{\hat{\lambda}}^{B}\left((I-\hat{\lambda} A)-\hat{\gamma} L^{*}(I-T) L\right)
$$

we know that $\hat{T}$ is $\frac{3+2 \hat{\gamma}\|L\|^{2}}{4}$-averaged and $F(\hat{T})=\Omega_{L, T}^{A+B}$.

Hence, for each $j \in \mathbb{N}$ we have

$$
\begin{aligned}
\left\|x_{n_{j}}-\hat{T} x_{n_{j}}\right\| & \leq\left\|x_{n_{j}}-u_{n_{j}}\right\|+\left\|T_{n_{j}} x_{n_{j}}-\hat{T} x_{n_{j}}\right\| \\
& \leq\left\|x_{n_{j}}-u_{n_{j}}\right\|+\left\|J_{\lambda_{n_{j}}}^{B} z_{j}-J_{\hat{\lambda}}^{B} z_{j}\right\|+\left\|J_{\hat{\lambda}}^{B} z_{j}-\hat{T} x_{n_{j}}\right\|,
\end{aligned}
$$

where $z_{j}=\left(\left(I-\lambda_{n_{j}} A\right)-\gamma_{n_{j}} L^{*}(I-T) L\right) x_{n_{j}}$. Now, we estimate the last term in (3.16). We have

$$
\begin{aligned}
\left\|J_{\hat{\lambda}}^{B} z_{j}-\hat{T} x_{n_{j}}\right\| & =\left\|J_{\hat{\lambda}}^{B}\left(\left(I-\lambda_{n_{j}} A\right)-\gamma_{n_{j}} L^{*}(I-T) L\right) x_{n_{j}}-J_{\hat{\lambda}}^{B}\left((I-\hat{\lambda} A)-\hat{\gamma} L^{*}(I-T) L\right) x_{n_{j}}\right\| \\
& \leq\left\|\left(\left(I-\lambda_{n_{j}} A\right)-\gamma_{n_{j}} L^{*}(I-T) L\right) x_{n_{j}}-\left((I-\hat{\lambda} A)-\hat{\gamma} L^{*}(I-T) L\right) x_{n_{j}}\right\| \\
& \leq\left\|\left(\lambda_{n_{j}}-\hat{\lambda}\right) A x_{n_{j}}\right\|+\left\|\left(\gamma_{n_{j}}-\hat{\gamma}\right) L^{*}(I-T) L x_{n_{j}}\right\| \\
& \leq\left|\lambda_{n_{j}}-\hat{\lambda}\right|\left\|A x_{n_{j}}\right\|+2\left|\gamma_{n_{j}}-\hat{\gamma}\right|\left\|L^{*}\right\|\|L\|\left\|x_{n_{j}}-p\right\|
\end{aligned}
$$

for each $j \in \mathbb{N}$. This implies that

$$
\lim _{j \rightarrow \infty}\left\|J_{\hat{\lambda}}^{B} z_{j}-\hat{T} x_{n_{j}}\right\|=0
$$


Next, we estimate the second term in (3.16). By Lemma 2.5, we have

$$
\begin{aligned}
\left\|J_{\lambda_{n_{j}}}^{B} z_{j}-J_{\hat{\lambda}}^{B} z_{j}\right\| & =\left\|J_{\hat{\lambda}}^{B}\left(\frac{\hat{\lambda}}{\lambda_{n_{j}}} z_{j}+\left(1-\frac{\hat{\lambda}}{\lambda_{n_{j}}}\right) J_{\lambda_{n_{j}}}^{B} z_{j}\right)-J_{\hat{\lambda}}^{B} z_{j}\right\| \\
& \leq\left\|\frac{\hat{\lambda}}{\lambda_{n_{j}}} z_{j}+\left(1-\frac{\hat{\lambda}}{\lambda_{n_{j}}}\right) J_{\lambda_{n_{j}}}^{B} z_{j}-z_{j}\right\| \\
& =\left\|\left(1-\frac{\hat{\lambda}}{\lambda_{n_{j}}}\right) J_{\lambda_{n_{j}}}^{B} z_{j}-\left(1-\frac{\hat{\lambda}}{\lambda_{n_{j}}}\right) z_{j}\right\| \\
& =\left\|\left(1-\frac{\hat{\lambda}}{\lambda_{n_{j}}}\right)\left(J_{\lambda_{n_{j}}}^{B} z_{j}-z_{j}\right)\right\| \\
& =\left|1-\frac{\hat{\lambda}}{\lambda_{n_{j}}}\right|\left\|J_{\lambda_{n_{j}}}^{B} z_{j}-z_{j}\right\|, \quad \forall j \geq 1 .
\end{aligned}
$$

Also for each $j \in \mathbb{N}$ we have

$$
\begin{aligned}
\left\|J_{\lambda_{n_{j}}}^{B} z_{j}-z_{j}\right\| & =\left\|T_{n_{j}} x_{n_{j}}-z_{j}\right\| \\
& =\left\|u_{n_{j}}-x_{n_{j}}+\lambda_{n_{j}} A x_{n_{j}}+\gamma_{n_{j}} L^{*}(I-T) L x_{n_{j}}\right\| \\
& \leq\left\|u_{n_{j}}-x_{n_{j}}\right\|+\lambda_{n_{j}}\left\|A x_{n_{j}}\right\|+\gamma_{n_{j}}\left\|L^{*}(I-T) L x_{n_{j}}\right\| \\
& \leq\left\|u_{n_{j}}-x_{n_{j}}\right\|+\lambda_{n_{j}}\left\|A x_{n_{j}}\right\|+2 \gamma_{n_{j}}\left\|L^{*}\right\|\|L\|\left\|x_{n_{j}}-p\right\| .
\end{aligned}
$$

This shows that $\left\{\left\|\left(J_{n_{j}}^{B} z_{j}-z_{j}\right)\right\|\right\}$ is a bounded sequence. This, together with (3.18), implies

$$
\lim _{j \rightarrow \infty}\left\|J_{\lambda_{n_{j}}}^{B} z_{j}-J_{\hat{\lambda}}^{B} z_{j}\right\|=0 .
$$

Substituting (3.14), (3.17) and (3.19) into (3.16), we get

$$
\lim _{j \rightarrow \infty}\left\|x_{n_{j}}-\hat{T} x_{n_{j}}\right\|=0
$$

Thus, by Lemma 2.6, it follows that $\hat{x} \in F(\hat{T})=\Omega_{L, T}^{A+B}$.

Furthermore, it follows from (3.13) and (3.14) that $\left\{u_{n}\right\},\left\{x_{n}\right\}$ and $\left\{S\left(u_{n}\right)\right\}$ have the same asymptotical behavior, so $\left\{u_{n}\right\}$ also converges weakly to $\hat{x}$. Since $S$ is nonexpansive, by (3.13) and Lemma 2.6, we obtain that $\hat{x} \in F(S)$. Thus $\hat{x} \in \Omega_{L, T}^{A+B} \cap F(S)$.

Next, we claim that

$$
\lim \sup _{n \rightarrow \infty}\left\langle f(z)-z, x_{n}-z\right\rangle \leq 0,
$$

where $z=P_{F(S) \cap \Omega_{L, T}^{A+B}} f(z)$.

Indeed, we have

$$
\begin{aligned}
\limsup _{n \rightarrow \infty}\left\langle f(z)-z, x_{n}-z\right\rangle & =\limsup _{n \rightarrow \infty}\left\langle f(z)-z, S u_{n}-z\right\rangle \\
& \leq \limsup _{n \rightarrow \infty}\left\langle f(z)-z, u_{n}-z\right\rangle
\end{aligned}
$$




$$
\begin{aligned}
& =\langle f(z)-z, \hat{x}-z\rangle \\
& \leq 0
\end{aligned}
$$

since $z=P_{F(S) \cap \Omega_{L, T}^{A+B}} f(z)$.

Finally, we show that $x_{n} \rightarrow z$. Indeed, we have

$$
\begin{aligned}
\left\|x_{n+1}-z\right\|^{2}= & \left\langle\alpha_{n} f\left(x_{n}\right)+\left(1-\alpha_{n}\right) S u_{n}-z, x_{n+1}-z\right\rangle \\
= & \alpha_{n}\left\langle f\left(x_{n}\right)-z, x_{n+1}-z\right\rangle+\left(1-\alpha_{n}\right)\left\langle S u_{n}-z, x_{n+1}-z\right\rangle \\
\leq & \alpha_{n}\left\langle f\left(x_{n}\right)-z, x_{n+1}-z\right\rangle+\left(1-\alpha_{n}\right)\left\langle u_{n}-z, x_{n+1}-z\right\rangle \\
\leq & \alpha_{n}\left\langle f\left(x_{n}\right)-f(z), x_{n+1}-z\right\rangle+\alpha_{n}\left\langle f(z)-z, x_{n+1}-z\right\rangle \\
& +\left(1-\alpha_{n}\right)\left\langle x_{n}-z, x_{n+1}-z\right\rangle \\
\leq & \frac{\alpha_{n}}{2}\left\{\|f(x)-f(z)\|^{2}+\left\|x_{n+1}-z\right\|^{2}\right\}+\alpha_{n}\left\langle f(z)-z, x_{n+1}-z\right\rangle \\
& +\frac{\left(1-\alpha_{n}\right)}{2}\left\{\left\|x_{n}-z\right\|^{2}+\left\|x_{n+1}-z\right\|^{2}\right\} \\
\leq & \frac{1}{2}\left(1-\alpha_{n}\left(1-\alpha^{2}\right)\right)\left\|x_{n}-z\right\|^{2}+\frac{\left(1-\alpha_{n}\right)}{2}\left\|x_{n+1}-z\right\|^{2} \\
& +\frac{\alpha_{n}}{2}\left\|x_{n+1}-z\right\|^{2}+\alpha_{n}\left\langle f(z)-z, x_{n+1}-z\right\rangle,
\end{aligned}
$$

which implies that

$$
\left\|x_{n+1}-z\right\|^{2} \leq\left(1-\alpha_{n}\left(1-\alpha^{2}\right)\right)\left\|x_{n}-z\right\|^{2}+2 \alpha_{n}\left(f(z)-z, x_{n+1}-z\right) .
$$

Now, by using (3.22) and Lemma 2.8, we deduce that $x_{n} \rightarrow z$. Further it follows from $\| u_{n}-$ $x_{n} \| \rightarrow 0, u_{n} \rightarrow \hat{x} \in F(S) \cap \Omega_{L, T}^{A+B}$ and $x_{n} \rightarrow z$ as $n \rightarrow \infty$, that $z=\hat{x}$. This completes the proof.

If $A:=0$, the zero operator, then the following result can be obtained from Theorem 3.2 immediately.

Corollary 3.3 Let $H_{1}$ and $H_{2}$ be Hilbert spaces. Let $B: H_{1} \rightarrow 2^{H_{1}}$ be a maximal monotone operator, $T: H_{2} \rightarrow H_{2}$ a nonexpansive mapping and $L: H_{1} \rightarrow H_{2}$ a bounded linear operator. Let $S: H_{1} \rightarrow H_{1}$ be a nonexpansive mapping such that $\Gamma=F(S) \cap B^{-1}(0) \cap L^{-1}(F(T)) \neq$ $\emptyset$. Let $f: H_{1} \rightarrow H_{1}$ be a contraction mapping with a contractive constant $\alpha \in(0,1)$. For any given $x_{0} \in H_{1}$, let $\left\{u_{n}\right\}$ and $\left\{x_{n}\right\}$ be the sequences generated by

$$
\left\{\begin{array}{l}
u_{n}=J_{\lambda_{n}}^{B}\left(\left(I-\gamma_{n} L^{*}(I-T) L\right) x_{n}, \quad \forall n \geq 0 .\right. \\
x_{n+1}=\alpha_{n} f\left(x_{n}\right)+\left(1-\alpha_{n}\right) S u_{n},
\end{array}\right.
$$

If the sequences $\left\{\alpha_{n}\right\},\left\{\lambda_{n}\right\}$ and $\left\{\gamma_{n}\right\}$ satisfy all the conditions in Theorem 3.2, then the sequences $\left\{u_{n}\right\}$ and $\left\{x_{n}\right\}$ both converge strongly to $z=P_{\Gamma} f(z)$ which is a solution of problem (1.5) with $A=0$.

If $H_{1}=H_{2}, L=I$, then by applying Theorem 3.2, we can obtain the following result. 
Corollary 3.4 Let $H_{1}$ be Hilbert spaces. Let $A: H_{1} \rightarrow H_{1}$ be a $\beta$-ism and $B: H_{1} \rightarrow 2^{H_{1}}$ be a maximal monotone operator. Let $S: H_{1} \rightarrow H_{1}$ be a nonexpansive mapping such that $\Gamma_{1}=F(S) \cap(A+B)^{-1} 0 \cap F(T) \neq \emptyset$. Let $f: H_{1} \rightarrow H_{1}$ be a contraction mapping with constant $\alpha \in(0,1)$. For any $x_{0} \in H_{1}$ arbitrarily, let the iterative sequences $\left\{u_{n}\right\}$ and $\left\{x_{n}\right\}$ be generated by

$$
x_{n+1}=\alpha_{n} f\left(x_{n}\right)+\left(1-\alpha_{n}\right) S J_{\lambda_{n}}^{B}\left(\left(I-\lambda_{n} A\right)-\gamma_{n}(I-T)\right) x_{n} .
$$

If the sequences $\left\{\alpha_{n}\right\},\left\{\lambda_{n}\right\}$ and $\left\{\gamma_{n}\right\}$ satisfy all the conditions in Theorem 3.2, then the sequences $\left\{u_{n}\right\}$ and $\left\{x_{n}\right\}$ both converge strongly to $z \in \Gamma_{1}$, where $z=P_{\Gamma_{1}} f(z)$.

\section{Applications}

In this section, we will utilize the results presented in the paper to study variational inequality problems, convex minimization problem and split common fixed point problem in Hilbert spaces.

\subsection{Application to variational inequality problem}

Let $C$ be a nonempty closed and convex subset of a Hilbert space $H$. Recall that the normal cone to $C$ at $u \in C$ is defined by

$$
N_{C}(u)=\{z \in H:\langle z, y-u\rangle \leq 0, \forall y \in C\} .
$$

It is well known that $N_{C}$ is a maximal monotone operator. In the case $B:=N_{C}: H \rightarrow 2^{H}$ we can verify that the problem of finding $x^{*} \in H$ such that $0 \in A x^{*}+B x^{*}$ is reduced to the problem of finding $x^{*} \in C$ such that

$$
\left\langle A x^{*}, x-x^{*}\right\rangle \geq 0, \quad \forall x \in C .
$$

In the sequel, we denote by $\operatorname{VIP}(C, A)$ the solution set of problem (4.1). In this case, we also have $J_{\lambda}^{B}=P_{C}$ (the metric projection of $H$ onto $C$ ). By the above consideration, problem (1.5) is reduced to finding

$$
x^{*} \in \operatorname{VIP}(C, A) \quad \text { such that } L x^{*} \in F(T) \text { and } x^{*} \in F(S) .
$$

Therefore, the following convergence theorem can be immediately obtained from Theorem 3.2.

Theorem 4.1 Let $H_{1}$ and $H_{2}$ be Hilbert spaces. Let $A: H_{1} \rightarrow H_{1}$ be a $\beta$-ism operator, $T: H_{2} \rightarrow H_{2}$ a nonexpansive mapping and $L: H_{1} \rightarrow H_{2}$ a bounded linear operator. Let $S: H_{1} \rightarrow H_{1}$ be a nonexpansive mapping such that $F(S) \cap \Omega_{L, T}^{A, C} \neq \emptyset$, where

$$
\Omega_{L, T}^{A, C}:=\operatorname{VIP}(C, A) \cap L^{-1}(F(T)) .
$$

Let $f: H_{1} \rightarrow H_{1}$ be a contraction mapping with a contractive constant $\alpha \in(0,1)$. For any given $x_{0} \in H_{1}$, let the sequences $\left\{u_{n}\right\}$ and $\left\{x_{n}\right\}$ be generated by

$$
\left\{\begin{array}{l}
u_{n}=P_{C}\left(\left(I-\lambda_{n} A\right)-\gamma_{n} L^{*}(I-T) L\right) x_{n}, \\
x_{n+1}=\alpha_{n} f\left(x_{n}\right)+\left(1-\alpha_{n}\right) S u_{n},
\end{array}\right.
$$


where $\left\{\alpha_{n}\right\}$ is a sequence in $(0,1)$ such that $\lim _{n \rightarrow \infty} \alpha_{n}=0, \sum_{n=0}^{\infty} \alpha_{n}=\infty, \sum_{n=1}^{\infty}\left|\alpha_{n}-\alpha_{n-1}\right|<$ $\infty, L^{*}$ is the adjoint of $L$, and the sequences $\left\{\lambda_{n}\right\}$ and $\left\{\gamma_{n}\right\}$ satisfy conditions $(i)-(i i)$ in Theorem 3.2. Then the sequences $\left\{u_{n}\right\}$ and $\left\{x_{n}\right\}$ both converge strongly to $z=P_{F(S) \cap \Omega_{L, T}^{A, C}} f(z)$, which is a solution of problem (4.2).

\subsection{Application to convex minimization problem}

Let $g: H \rightarrow R$ be a convex function, which is also Fréchet differentiable. Let $C$ be a given closed convex subset of $H$. In this case, by setting $A:=\nabla g$, the gradient of $g$, and $B=N_{C}$, the problem of finding $x^{*} \in(A+B)^{-1} 0$ is equivalent to finding a point $x^{*} \in C$ such that

$$
\left\langle\nabla g\left(x^{*}\right), x-x^{*}\right\rangle \geq 0, \quad \forall x \in C
$$

Note that (4.4) is equivalent to the following minimization problem: find $x^{*} \in C$ such that

$$
x^{*} \in \arg \min _{x \in C} g(x) .
$$

Thus, in this situation, problem (1.5) is reduced to the problem of finding

$$
x^{*} \in \arg \min _{x \in C} g(x) \quad \text { such that } L x^{*} \in F(T) \text { and } x^{*} \in F(S) \text {. }
$$

Denote by

$$
\Omega_{L, T}^{g, C}:=\arg \min _{x \in C} g(x) \cap L^{-1}(F(T)) .
$$

Then, by using Theorem 3.2, we can obtain the following result.

Theorem 4.2 Let $H_{1}$ and $H_{2}$ be Hilbert spaces and let $C$ be a nonempty closed convex subset of $H_{1}$. Let $g: H_{1} \rightarrow \mathbb{R}$ be a convex and Fréchet differentiable function, $\nabla g$ be $\beta$ Lipschitz, $T: H_{2} \rightarrow H_{2}$ be a nonexpansive mapping, and let $L: H_{1} \rightarrow H_{2}$ be a bounded linear operator. Let $S: H_{1} \rightarrow H_{1}$ be a nonexpansive mapping such that $F(S) \cap \Omega_{L, T}^{g, C} \neq \emptyset$. Let $f: H_{1} \rightarrow H_{1}$ be a contraction mapping with a contractive constant $\alpha \in(0,1)$. For any given $x_{0} \in H_{1}$, let $\left\{u_{n}\right\}$ and $\left\{x_{n}\right\}$ be the sequences generated by

$$
\left\{\begin{array}{l}
u_{n}=P_{C}\left(\left(I-\lambda_{n} \nabla g\right)-\gamma_{n} L^{*}(I-T) L\right) x_{n}, \\
x_{n+1}=\alpha_{n} f\left(x_{n}\right)+\left(1-\alpha_{n}\right) S u_{n},
\end{array} \quad \forall n \geq 0 .\right.
$$

If the sequences $\left\{\alpha_{n}\right\},\left\{\lambda_{n}\right\}$ and $\left\{\gamma_{n}\right\}$ satisfy all the conditions in Theorem 3.2, then the sequences $\left\{u_{n}\right\}$ and $\left\{x_{n}\right\}$ both converge strongly to $z \in F(S) \cap \Omega_{L, T}^{g, C}$, where $z=P_{F(S) \cap \Omega_{L, T}^{g, C}} f(z)$, which is a solution of problem (4.5).

Proof Note that if $g: H \rightarrow \mathbb{R}$ is convex and $\nabla g: H \rightarrow H$ is $\beta$-Lipschitz continuous for $\beta>0$ then $\nabla g$ is $\frac{1}{\beta}$-ism (see [24]). Thus, the required result can be obtained immediately from Theorem 3.2. 


\subsection{Application to split common fixed point problem}

Let $V: H_{1} \rightarrow H_{1}$ be a nonexpansive mapping. Then, by Lemma 2.1(iii), we know that $A:=I-V$ is $\frac{1}{2}$-ism. Furthermore, $A x^{*}=0$ if and only if $x^{*} \in F(V)$. Hence problem (1.5) can be reduced to the problem of finding

$$
x^{*} \in F(V) \quad \text { such that } L x^{*} \in F(T) \text { and } x^{*} \in F(S) \text {, }
$$

where $T: H_{2} \rightarrow H_{2}, L: H_{1} \rightarrow H_{2}$ and $S: H_{1} \rightarrow H_{1}$ are mappings as in Theorem 3.2.

This problem is called the split common fixed point problem (SCFP), and was studied by many authors (see [25-28], for example). By using Theorem 3.2, we can obtain the following result.

Theorem 4.3 Let $H_{1}$ and $H_{2}$ be Hilbert spaces. Let $V: H_{1} \rightarrow H_{1}$ and $T: H_{2} \rightarrow H_{2}$ be nonexpansive mappings and $L: H_{1} \rightarrow H_{2}$ a bounded linear operator. Let $S: H_{1} \rightarrow H_{1}$ be a nonexpansive mapping such that $F(S) \cap \Omega_{L, T}^{V} \neq \emptyset$, where

$$
\Omega_{L, T}^{V}:=F(V) \cap L^{-1}(F(S))
$$

Let $f: H_{1} \rightarrow H_{1}$ be a contraction mapping with a contractive constant $\alpha \in(0,1)$. For any given $x_{0} \in H_{1}$, let be $\left\{u_{n}\right\}$ and $\left\{x_{n}\right\}$ be the iterative sequences generated by

$$
\left\{\begin{array}{l}
u_{n}=\left(I-\lambda_{n}\right) x_{n}+\lambda_{n} V x_{n}-\gamma_{n} L^{*}(I-T) L x_{n}, \quad \forall n \geq 0, \\
x_{n+1}=\alpha_{n} f\left(x_{n}\right)+\left(1-\alpha_{n}\right) S u_{n},
\end{array}\right.
$$

where the sequences $\left\{\alpha_{n}\right\},\left\{\lambda_{n}\right\}$ and $\left\{\gamma_{n}\right\}$ satisfy all the conditions in Theorem 3.2. Then the sequences $\left\{u_{n}\right\}$ and $\left\{x_{n}\right\}$ both converge strongly to a point $z=P_{F(S) \cap \Omega_{L, T}^{V}} f(z)$, which is a solution of problem (4.7).

Proof We consider $B:=0$, the zero operator. The required result follows from the fact that the zero operator is monotone and continuous, hence it is maximal monotone. Moreover, in this case, we see that $J_{\lambda}^{B}$ is the identity operator on $H_{1}$, for each $\lambda>0$. Thus algorithm (3.4) reduces to (4.8), by setting $A:=I-V$ and $B:=0$.

\section{Acknowledgements}

The authors would like to express their thanks to the Editor and the Referees for their helpful comments.

\section{Funding}

The first author was supported by Scientific Research Fund of Sichuan Provincial Department of Science and Technology (2015JY0165), the second author was supported by Scientific Research Fund of Sichuan Provincial Education Department (16ZA0331) and the third author was supported by The Natural Science Foundation of China Medical University,

Taichung, Taiwan.

Availability of data and materials

Not applicable.

\section{Competing interests}

None of the authors have any competing interests in the manuscript.

Authors' contributions

All authors contributed equally and significantly in writing this article. All authors read and approved the final manuscript.

\section{Author details}

${ }^{1}$ Department of Mathematics, Yibin University, Yibin, China. ${ }^{2}$ Center for General Education, China Medical University,

Taichung, Taiwan. 


\section{Publisher's Note}

Springer Nature remains neutral with regard to jurisdictional claims in published maps and institutional affiliations.

\section{Received: 6 July 2018 Accepted: 14 October 2018 Published online: 23 October 2018}

\section{References}

1. Censor, Y., Elfving, T.: A multiprojection algorithm using Bregman projections in product space. Numer. Algorithms 8 , $221-239(1994)$

2. Byrne, C.: Iterative oblique projection onto convex sets and the split feasibility problem. Inverse Probl. 18, 441-453 (2002)

3. Censor, Y., Bortfeld, T., Martin, B., Trofimov, A.: A unified approach for inversion problems in intensity-modulated radiation therapy. Phys. Med. Biol. 51, 2353-2365 (2006)

4. Martinet, B.: Régularisation dinéquations variationnelles par approximations successives. Rev. Fr. Inform. Rech. Opér. 3, 154-158 (1970)

5. Bruck, R.E., Reich, S.: Nonexpansive projections and resolvents of accretive operators in Banach spaces. Houst. J. Math. 3, 459-470 (1977)

6. Eckstein, J., Bertsckas, D.P.: On the Douglas-Rachford splitting method and the proximal point algorithm for maximal monotone operators. Math. Program. 55, 293-318 (1992)

7. Marino, G., Xu, H.K.: Convergence of generalized proximal point algorithm. Commun. Pure Appl. Anal. 3, 791-808 (2004)

8. Xu, H.K.: Iterative algorithms for nonlinear operators. J. Lond. Math. Soc. 66, 240-256 (2002)

9. Yao, Y., Noor, M.A.: On convergence criteria of generalized proximal point algorithms. J. Comput. Appl. Math. 217, 46-55 (2008)

10. Montira, S., Narin, P., Suthep, S.: Weak convergence theorems for split feasibility problems on zeros of the sum of monotone operators and fixed point sets in Hilbert spaces. Fixed Point Theory Appl. 2017, Article ID 6 (2017)

11. Byrne, C., Censor, Y., Gibali, A., Reich, S.: Weak and strong convergence of algorithms for the split common null point problem. J. Nonlinear Convex Anal. 13, 759-775 (2012)

12. Takahashi, W., Xu, H.K., Yao, J.C.: Iterative methods for generalized split feasibility problems in Hilbert spaces. Set-Valued Var. Anal. 23, 205-221 (2015)

13. Passty, G.B.: Ergodic convergence to a zero of the sum of monotone operators in Hilbert space. J. Math. Anal. Appl. 72, 383-390 (1979)

14. Baillon, J.B., Bruck, R.E., Reich, S.: On the asymptotic behavior of nonexpansive mappings and semigroups in Banach spaces. Houst. J. Math. 4, 1-9 (1978)

15. Boikanyo, O.A.: The viscosity approximation forward-backward splitting method for zeros of the sum of monotone operators. Abstr. Appl. Anal. 2016, Article ID 2371857 (2016)

16. Xu, H.K.: Averaged mappings and the gradient-projection algorithm. J. Optim. Theory Appl. 150, 360-378 (2011)

17. Byrne, C:: A unified treatment of some iterative algorithms in signal processing and image reconstruction. Inverse Probl. 20, 103-120 (2004)

18. Goebel, K., Reich, S.: Uniform Convexity, Hyperbolic Geometry, and Nonexpansive Mappings. Dekker, New York (1984)

19. Takahashi, W.: Introduction to Nonlinear and Convex Analysis. Yokohama Publishers, Yokohama (2009)

20. Takahashi, S., Takahashi, W., Toyoda, M.: Strong convergence theorems for maximal monotone operators with nonlinear mappings in Hilbert spaces. J. Optim. Theory Appl. 147, 27-41 (2010)

21. Barbu, V:: Nonlinear Semigroups and Differential Equations in Banach Spaces. Noordhoff, Leiden (1976)

22. Takahashi, W.: Nonlinear Functional Analysis: Fixed Point Theory and Its Applications. Yokohama Publishers, Yokohama (2000)

23. Xu, H.K.: Viscosity approximation methods for nonexpansive mapping. J. Math. Anal. Appl. 298, 279-291 (2004)

24. Baillon, J.B., Haddad, G.: Quelques propriétés des opérateurs angle-bornés et $n$-cycliquement monotones. Isr. J. Math. 26(2), 137-150 (1977)

25. Cui, H., Wang, F.: Iterative methods for the split common fixed point problem in Hilbert spaces. Fixed Point Theory Appl. 2014, Article ID 78 (2014)

26. Moudafi, A.: A note on the split common fixed-point problem for quasi-nonexpansive operators. Nonlinear Anal., Theory Methods Appl. 74, 4083-4087 (2011)

27. Shimizu, T., Takahashi, W.: Strong convergence to common fixed points of families of nonexpansive mappings. J. Math. Anal. Appl. 211, 71-83 (1997)

28. Zhao, J., He, S.: Strong convergence of the viscosity approximation process for the split common fixed-point problem of quasi-nonexpansive mappings. J. Appl. Math. 2012, Article ID 438023 (2012) 